\title{
Structural and lectin-detectable changes of liver induced by a long-term administration of antihistamine agent Loratadine
}

\author{
Olga Dudok, Alexander Lutsyk
}

Department of Histology, Cytology and Embryology, Danylo Halytsky Lviv National Medical University, Ukraine

\begin{abstract}
Adverse effects of pharmacological agents are currently under considerable attention of theoretical and clinical medicine. The aim of present investigation was to study in experiment the effect of a long-term administration of antihistamine drug Loratadine on the micromorphology and carbohydrate determinants of the liver. Experimental rats once a day during 30 days intragastrically received Loratadine in the form of an aqueous suspension in the dose of $0.15 \mathrm{mg} / \mathrm{kg}$ body weight. On days 10,30, 40, 50, 60 of experiment euthanasia was carried out, liver samples excised, fixed in $4 \%$ formaline and embedded in paraffin. Examination of haematoxylin and eosin stained sections revealed extension of sinusoid capillaries and central veins, granular dystrophy of hepatocytes supplemented with perivascular lymphoid infiltration, these phenomena receiving highest development on days 30th and 40th of experiment. At the same times PAS reaction demonstrated increased content of glycogen deposits in hepatocytes, apparently encompassing alterations in glucose metabolism. On the later stages of experiment (days 20th and 30th after the last Loratadine injection) signs of hepatocyte alteration decreased significantly, and content of glycogen returned to normal values. Loratadine administration induced the accumulation in the intra- and 2 perisinusoidal space of hepatic lobules of activated Kupffer cells, which were strongly PAS- and WGA-positive, as well as the enhanced exposure of DGIcNAc and DGalNAc determinants by glycoconjugates in within the altered hepatocytes. Our results indicate certain destructive effect of Loratadine on hepatic micromorphology and function; the cessation of this drug administration was accompanied by simultaneous strengthening of regenerative processes.
\end{abstract}

Keywords: rat liver histology, lectin histochemistry, H1-blocker Loratadine.

\section{Introduction}

Nowadays more than 63 thousand chemical compounds are used by modern civilization, 55 thousand of which, including different kind pharmacological agents, can be dangerous to human health. Every year more than 1 million people suffer from the side effects of pharmacotherapy, resulting economic losses estimated as 136 billion dollars [1]. Since liver takes central position in the metabolization of xenobiotics and drugs, this organ most oftenly is subjected to injuries $[2,3]$. The leading mechanisms of these lesions development is direct action of a pharmacological agent or of its reactive metabolites on the liver cells that leads to their death by necrosis or induction of apoptosis [4, 5].

Recently it was reported of possible toxic effects on the human organism of antihistamine agents, which are widely applied in the treatment of patients with various allergic conditions [6]. Patients are oftenly using these drugs without consulting a doctor. It was documented the use of antihistamines as components of drug mixtures and acute poisoning by them of drug-abusers [7]. 
Moreover, investigations are conducted on possible influence of such drugs on the health of employees in pharmaceutical industry, directly involved in their production [8].

In the available literature we find no data on possible hepatotoxic effect of antihistamines leading to the structural reorganization of liver, including its carbohydrate determinants. Meanwhile, their lectin histochemistry investigation may be of practical interest due to important role played by cell surface, cytoplasmic and 3 extracellular glycoconjugates in normal histophysiology; carbohydrate determinants are among the first to be damaged during pathological lesions development [9-12]. Therefore the aim of present investigation was to study long-term influences of Loratadine - H1-histamine receptor blocker - on hepatic tissues micromorphology and lectin receptor sites.

\section{Material and methods}

Experiments were carried out on 60 mature male Vistar rats 160-200 g of weight, kept under standard conditions of vivarium. All manipulations were performed in accordance with the provisions of the European Convention for the protection of vertebrate animals used for experimental and other scientific purposes (Strasbourg, 1986) and the law of Ukraine "About protection of animals from cruel treatment" (No 1759-VI of 15.12.2009).

Animals were subdivided into two groups: the first group (20 rats) served as a control; second group animals $(n=40)$ once a day during 30 days intragastrically received Loratadine in the form of an aqueous suspension in the dose of $0.15 \mathrm{mg} / \mathrm{kg}$ of body weight. Loratadine is a second generation $\mathrm{H} 1$-histamine receptors blocker, chemically designated as "Ethyl ether -4-8-chloro-5,6-dihydro-11H-benzo-[5,6]cyclohepta[1,2-b]pyridin-11-len)-1-piridincarbonic acid", produced by "FARMACHEM SA Chem Limited" (India). The administered dose corresponded to the average daily therapeutic dose for humans. On 10th and 30th days of drug administration, and on days 40s, 50s and 60s from the beginning of experiment, animals were subjected to euthanasia with subsequent dislocation of cervical vertebrae; thereafter liver samples were collected for morphological studies.

For routine histological examination obtained material was fixed in $4 \%$ neutral formalin and embedded in paraffin; sections 5-7 $\mu \mathrm{m}$ thick were stained with haematoxylin and eosin. For getting semi-thin sections tissue samples were fixed in $2 \%$ OsO4, dehydrated and embedded into epon-araldite; $1 \mu \mathrm{m}$ sections were cut using ultratome YMPT-3M, subjected to PAS reaction and counterstained with methylene 4 blue [13].

Carbohydrate determinants of hepatic tissues were detected by a set of 7 lectins (Table 1). All used lectins were purified and coupled to horseradish peroxidase in the laboratory by Dr. Pharm. Sci. V.O.Antonyuk. Lectin receptor sites were visualized in PBS, containing $0.05 \%$ diaminobenzidine (Sigma, St.Louis, USA) and 0.015\% $\mathrm{H} 2 \mathrm{O} 2$ as described elsewhere [14]. Slides were studied and pictures taken using Leica DM 2500 microscope equipped with Leica DFC 450C digital camera.

\section{Results}

Examination of control specimens stained with haematoxylin and eosin revealed typical rat liver micromorphology (Figure 1), in comparison to which liver of Loratadine treated animals exposed certain signs characteristic for drug metabolization. Namely, on the experimental day 30th sinusoid capillaries expansion was accompanied with periportal lymphoid infiltration (Figure 1B). On the day 40th increased density of hepatocytes cytoplasm and nuclei was associated with decomplication of hepatic plates and sludge phenomena in within the expanded sinusoids (Figure 1C). Hepatocytes of the same group animals exposed signs of granular degeneration, hydropic dystrophy and nuclear pyknosis (Figure 1D).

Table 1. Lectins used and their carbohydrate specificity, according to data of V.0. Antonyuk [15]

\begin{tabular}{|c|c|c|c|}
\hline No & Lectin name and abbreviation & Carbohydrate specificity & Complementary oligo-/ polysaccharide \\
\hline 1 & Peanut agglutinin, PNA & BDGal(1-3)DGalNAc & Thomsen-Friedenreich antigen \\
\hline 2 & Laburnum anagyroides bark agglutinin, LABA & LFuc & Gal( $(\beta 1-4) F u c(\beta 1-3) G l c$ \\
\hline 3 & Sambucus nigra agglutinin, SNA & NeuNAc( $\alpha 2-6)$ DGal & NeuNAc( $\alpha 2-6) \operatorname{Gal}(\beta 1-4) \operatorname{GlcNAc}(\beta 1-2)$ \\
\hline 4 & Wheat germ agglutinin, WGA & DGlcNAc $>$ NeuNAc & 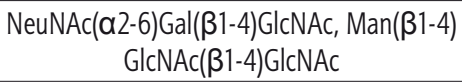 \\
\hline 5 & Galanthus nivalis agglutinin, GNA & aDMan & $\operatorname{Man}(\alpha 1-3) \operatorname{Man}(\beta 1-4) G \mid c N A c$ \\
\hline 6 & Clitocybe nebularis fungus agglutinin, CNFA & DGalNAc( $(\beta 1-4) G I c N A c$ & Not estimated \\
\hline 7 & Helix pomatia agglutinin, HPA & aDGalNAc & GalNAc(a1-3)GalNAc \\
\hline
\end{tabular}



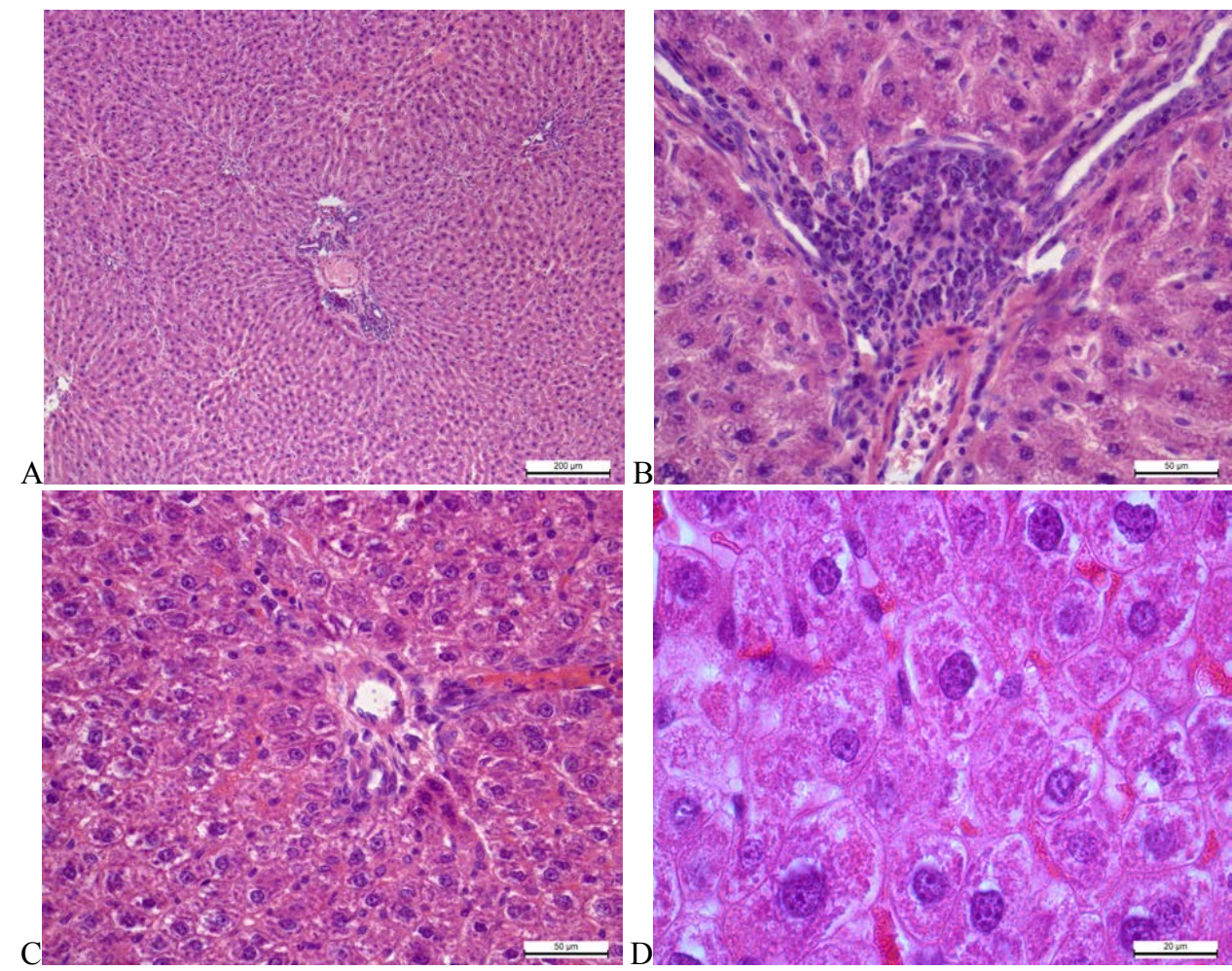

Figure 1. Changes in rat liver micromorphology induced by a long-term Loratadine administration. A. Liver of intact rat; B. Experimental day 30th: perivascular lymphoid infiltration; C. Experimental day 40th : extension of sinusoid capillaries, hepatic plates decomplication; D. Hepatocytes on the experimental day 40th: signs of granular degeneration, hydropic dystrophy and nuclear pyknosis. Haematoxylin and eosin, original magnification $x 100(A), x 400(B, C), x 1000(D)$
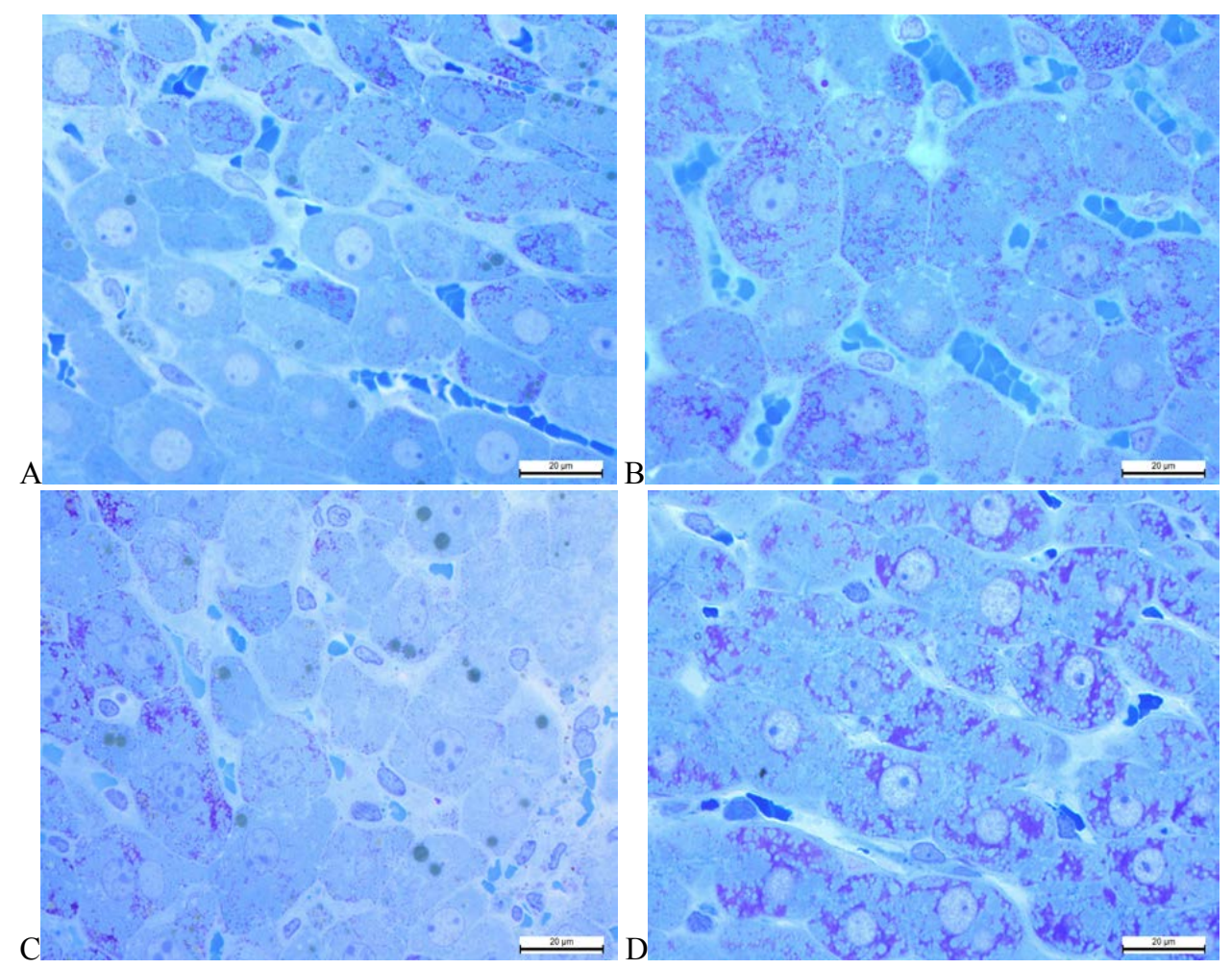

Figure 2. Semi-thin sections, PAS-reaction with methylene blue counterstaining. A. Liver of intact rat: dark blue coloration of small size Kupffer cells with intra- and perisinusoidal localization. B. 30th day of Loratadine treatment: accumulation of fine glycogen granules in the cytoplasm of hepatocytes. C. Experimental day 40th: densely packed glycogen inclusion overload groups of dystrophic hepatocytes; D. Experimental day 60s: amount of glycogen inclusions close to normal, intra- and perisinusoidal accumulation of Kupffer cells. Original magnification x1000 (A-D) 
Semi-thin sections of liver samples of control rats, as well as of experimental animals at early stages of Loratadine treatment demonstrated typical polygonal shape hepatocytes, endotheliocyte lined sinusoids, with few small densely stained cells with intra- and perisinusoidal localization - apparently Kupffer and Ito cells (Figure 2A). On the experimental day 30th the accumulation of fine glycogen granules was detected in the hepatocytes (Figure 2B); on day 40th these densely packed glycogen inclusions overloaded groups of hepatocytes, apparently encompassing their granular dystrophy (Figure 2C). Up to experimental day 60s the amount of glycogen inclusions decreased significantly, while number of Kupffer and Ito cells in within the hepatic lobules increased (Figure 2D).

Used lectins showed rather differential binding to carbohydrate determinants of 5 hepatic tissues in control and experimental animals. Most informative results were obtained with WGA, CNFA and HPA. Namely, in control rats WGA strongly labeled endothelial cell lining of liver sinusoids, cytoplasm and nuclei of hepatocytes being completely non reactive (Figure $3 \mathrm{~A}$ ). After 10 days of Loratadine administration this lectin reactivity was detected in the cytoplasm of small cells with periportal localization (Figure 3B). Number of these cells increased in intra- and perisinusoidal spaces on days 30th of experiment; similarly increased the exposure of WGA receptor sites within the cytoplasm of hepatocytes (Figure 3C, D).

CNFA in the liver of control rats selectively labeled bile capillaries and plasma membranes of hepatocytes, other hepatic elements being areactive (Figure 4A). In the liver of early stage experimental animals this lectin binding was restricted to cytoplasmic and nuclear glycoconjugates of hepatocytes and central veins endothelium (Figure 4B). On experimental days 40s CNFA binding was additionally detected within the cells of intra- and perisinusoidal localization, apparently activated Kupffer and Ito cells (Figure 4C). On days 60 s nuclei and plasma membrane of hepatocytes demonstrated enhanced lectin reactivity (Figure 4D).

HPA binding in the liver of control rats was restricted mostly to the luminal surface of sinusoid capillaries and plasma membranes of hepatocytes (Figure 5A). Among the most interesting findings was this lectin strong reactivity with cytoplasmic glycoconjugates of plasma cells (Figure 5B), which were previously identified in within the hepatic lobules by means of electron microscopy [16]. Loratadine administration induced significant accumulation of HPA receptor sites in the
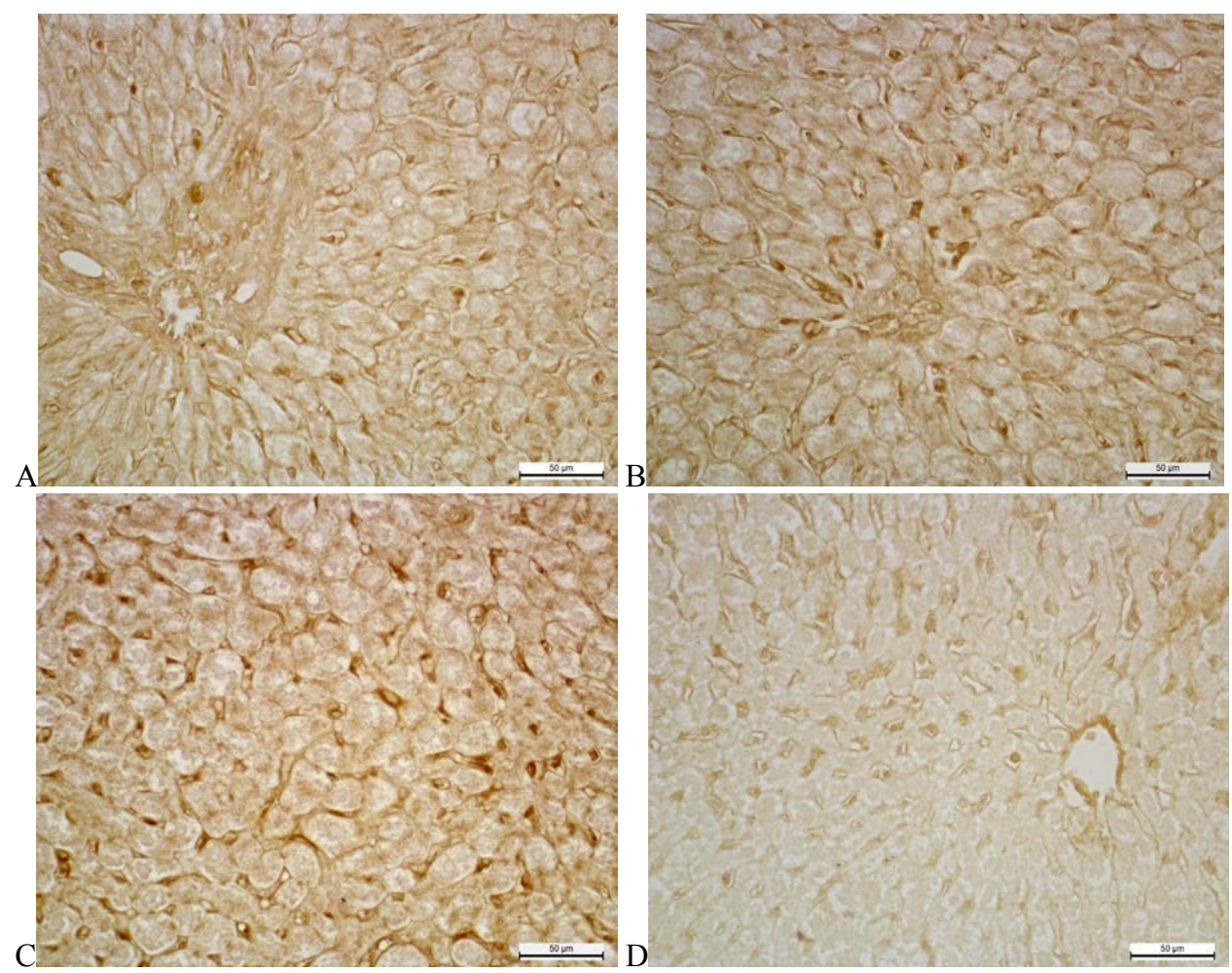

Figure 3. Redistribution of WGA receptor sites after Loratadine administration: liver of intact rat (A), on experimental days 10th (B), 40s (C), and 50s (D). Lectin label restricted to few Kupffer cells in periportal area (A), surrounding central vein (B); increased number of strong WGA-positive cells with intraand perisinusoidal localization (C, D); enhanced reactivity of hepatocytes (C) and of central vein endothelium (D). Original magnification x400 (A-D) 

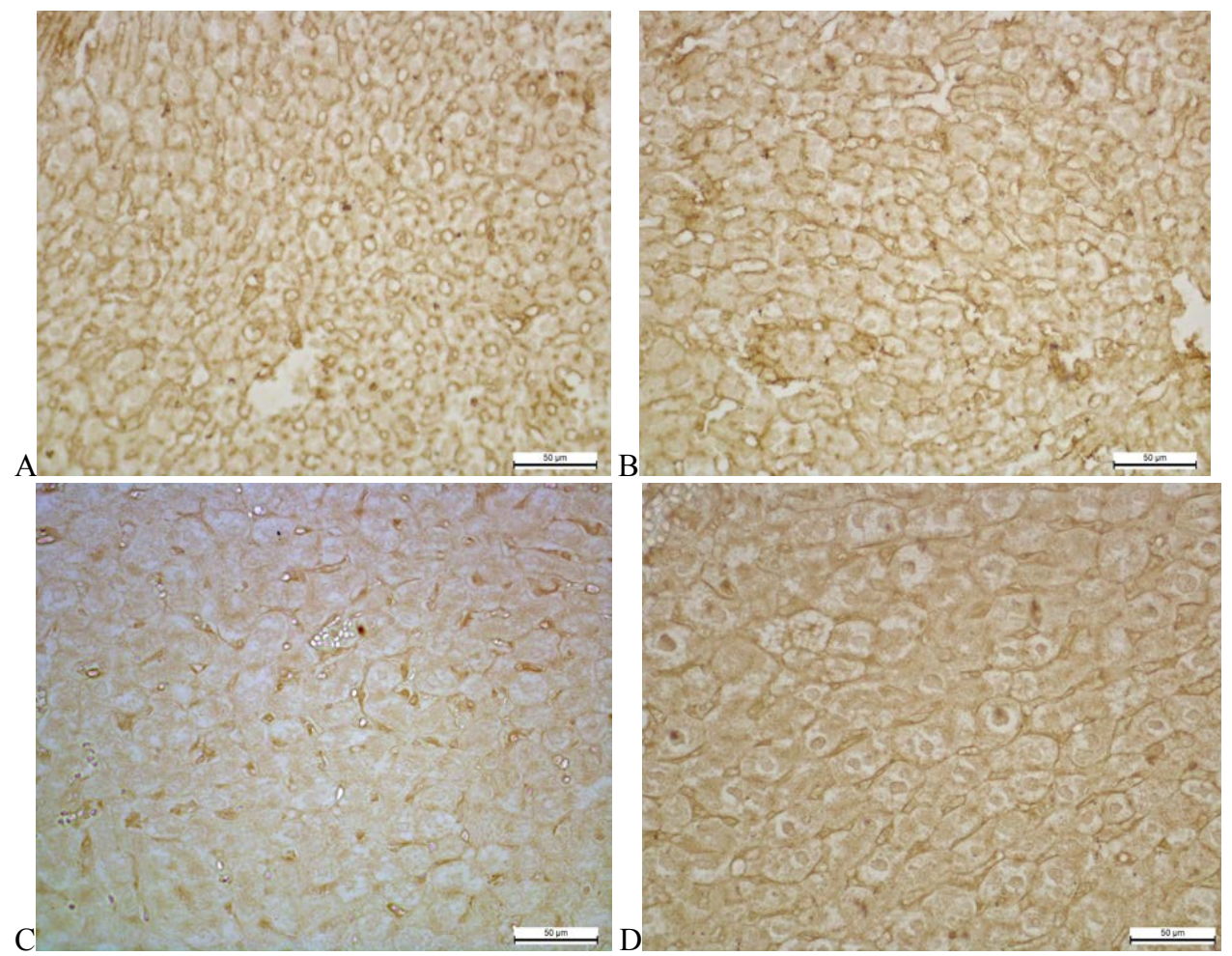

Figure 4. CNFA binding to liver of intact rat in comparison to liver after different terms of Loratadine administration. A. Liver of intact rat: lectin labeling of bile canaliculi and plasma membranes of hepatocytes. B. Experimental day 10th: enhanced reactivity of hepatocytes nuclei. C. 40s day of experiment: reduced reactivity of hepatocytes cytoplasm, bile canaliculi strong positive. D. 60s day of experiment: increased reactivity of hepatocytes cytoplasmic and nuclear glycoconjugates. Original magnification x400 (A-D)
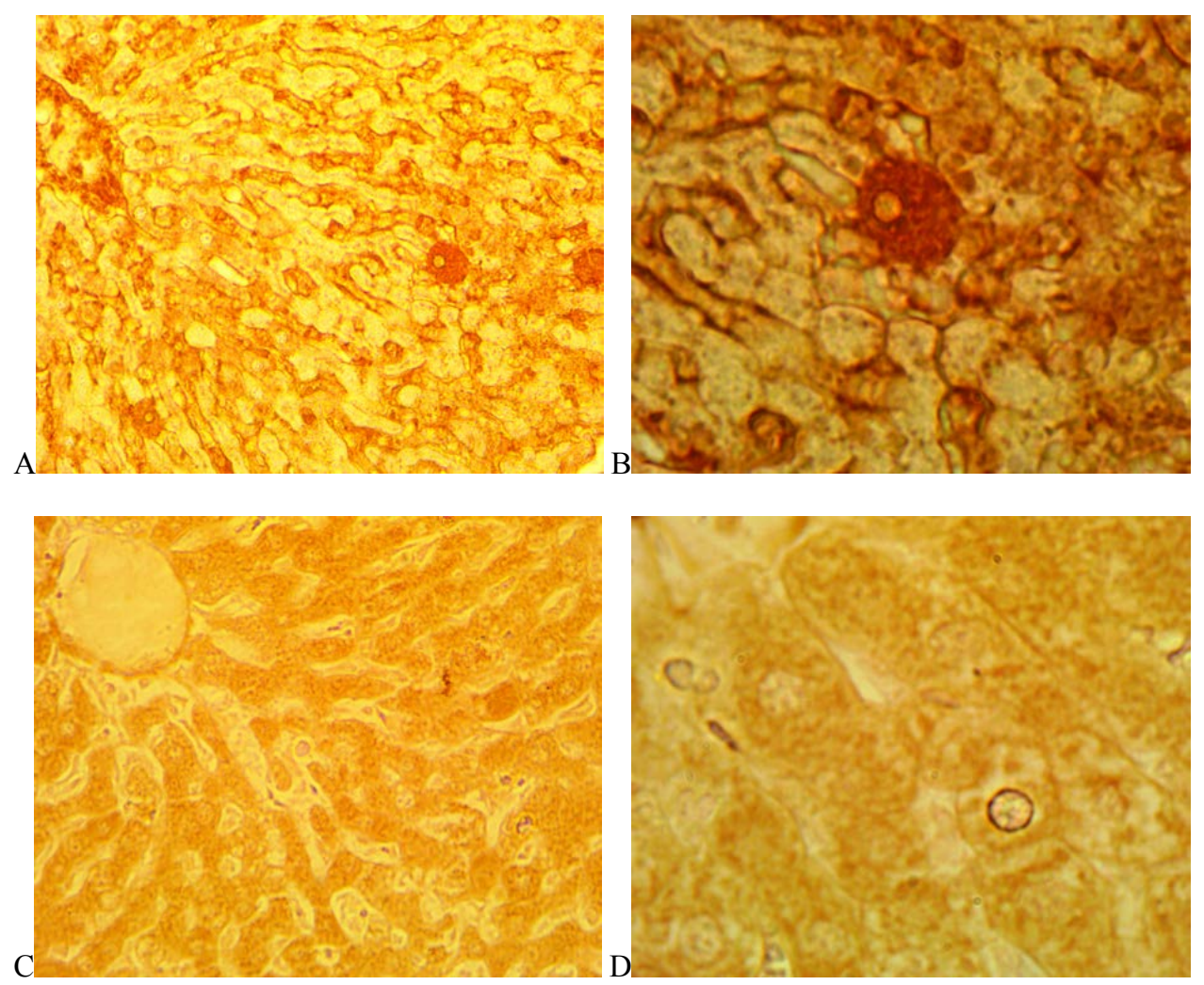

Figure 5. HPA label in hepatic tissue of control rat $(A, B)$ and its redistribution on day 30th of Loratadine treatment $(C, D)$. A. Intense reactivity of central vein endothelium, sinusoid capillaries, and cytoplasmic glycoconjugates of few plasma cells. B. Plasma cell in between plates of hepatocytes. C, D. Enhanced reactivity of cytoplsmic glycoconjugates in within the hepatocytes. Original magnification x400 (A, C), x1000 (B, D) 
cytoplasm of hepatocytes, this redistribution being especially prominent on day 30th of experiment (Figure $5 \mathrm{C}, \mathrm{D}$ ). With the rest of lectins used (PNA, GNA, SNA and LABA) no remarkable differences were found in the labeling of liver structures in between control and Loratadine-treated rats.

\section{Discussion}

Our results demonstrate that long-term daily administration of Loratadine induce 6 changes in liver micromorphology, highest manifestations of which account for 30th-40s days of experiment. These changes gradually decrease up to experimental day 60s (till the 30th day after last Loratadine administration). Detected signs of granular, and, in parts, hydropic dystrophy, apparently encompass transient rearrangement of biosynthetic function of hepatocytes into metabolic function - a phenomenon reported by Yvashkin et al. [17], directed towards Loratadine metabolisation. Lymphoid infiltration of perivascular spaces most likely is indicative of antigenic stimulation in response to spontaneous conjugation of Loratadine metabolisation products with endogenous proteins resulting with the formation of haptens [18].

Rather unexpected was our finding on the ability of Loratadine to induce the accumulation of glycogen in hepatocytes. Dual interpretation of this phenomenon is possible: (1) some reactive metabolites of Loratadine can serve as triggers of glucose metabolic processes impairments, directing them towards enhancement of gluconeogenesis (this mechanism was reported by Yakovleva et al. [19] for metabolisation of xenobiotics); (2) a long-term blockade of H1-histamine receptors in hepatocytes (presence of which was documented by Camelo-Nunes [20]) affects the activity of intracellular enzymes (adenilatcyclase, cAMP), involved in the regulation of gluconeogenesis. The important role of these enzymes in the regulation of glucose metabolism under the influence of antihistamines was reported by Berezhnaya et al. [21].

On the experimental days $50 \mathrm{~s}$ and $60 \mathrm{~s}$ it was detected significant decrease of dystrophic changes in hepatocytes accompanied with the accumulation of Kupffer and Ito cells in within hepatic lobules. These observations apparently encompass reduction of the alterative processes with simultaneous strengthening of the regenerative mechanisms. In particular, according to Berezhnaya et al. [21] and Takeishi et al. [22], Kupffer cells stimulate regeneration of hepatocytes by enhanced expression of hepatocyte growth factor via
TNF2-independent mechanism, while cells of Ito are stimulating neovasculogenesis in clusters of regenerated hepatocytes [23].

Our findings on lectin reactivity of hepatic tissues are consistent and extend our previous observations on the redistribution of liver carbohydrates in streptozotocin-induced diabetes mellitus [24]. We assume that detected accumulation of WGA, CNFA and HPA receptor sites (DGICNAc and DGalNAc determinants) within the hepatocytes apparently encompass transient Loratadine induced alterations of these cells synthetic machinery. Among possible mechanisms causing this phenomenon can be inhibitory effect of used antihistamine drug on carbohydrates final glycosylation steps in within Golgi complex, or inhibition of glycoconjugates exocytosis via plasma membrane of affected hepatocytes.

Lectins proved their usefulness as selective histochemical markers of normal rat liver structures: WGA of vascular endothelium; CNFA - of bile canaliculi and plasma membranes of hepatocytes; HPA - of hepatic plasma cells. After Loratadine administration WGA intensely labeled cells with intra- and perisinusoidal localization - apparently activated Kupffer cells. Our recent results somewhat disagree with earlier observations of Yashchenko et al. [24] on strong PNA reactivity of rat Kupffer cells; however, these discrepancies should be investigated in future research.

\section{Conclusions}

Our studies demonstrate that prolonged use of $\mathrm{H} 1$-histamine receptors blocker Loratadine induce transient changes, which reflect shifting of hepatocytes biosynthetic activity to drug metabolisation. Although causing granular and, partly, hydropic dystrophy, as well as the accumulation of glycogen deposits in hepatocytes, detected changes do not lead ultimately to these cells necrosis or apoptosis. However, the drug can be potentially dangerous with concomitant liver disease (HCV and HBV, HIV infections, etc.). Loratadine administration induced redistribution of WGA, CNFA and HPA receptor sites within the hepatocytes (exposure of DGICNAc and DGalNAc determinants), apparently encompassing alterations of these cells carbohydrate synthetic machinery. Development of antigenic stimulation was reflected by the lymphoid infiltration of periportal areas, accumulation of activated 8 Kupffer and Ito cells in intra- and perisinusoidal spaces. Lectins can be recommended for selective histochemical labeling of rat liver structures: WGA - 
of vascular endothelium, activated Kupffer cells; CNFA - of bile canaliculi; HPA - of hepatic plasma cells.

\section{Acknowledgements}

The authors wish to thank Dr. Pharm. Sci. V.O. Antonyuk for providing lectin-peroxidase conjugates used in this investigation.

\section{Conflict of interest statement}

The authors declare no conflict of interest.

\section{Funding sources}

There are no sources of funding to declare.

\section{References}

1. Navaro VS, Senior JR. Drug-related hepatotoxicity. N Eng J Med. 2006;354(7):731-739.

2. Holt MP. Mechanisms of drug-induced liver injury. American Association of Pharmaceutical Scientists Journal. 2006;8(1):48-54

3. Bjorrnsson E, Olsson R. Suspected drug-induced liver fatalities reported to the WHO database. Dig Liver Dis. 2006;38:33-38.

4. William M, Lee MD. Drug-induced hepatotoxicity. N Eng J Med. 2003;349:474-485.

5. Malhi H, Gores GJ, Lemaster JJ. Apoptosis and necrosis in the liver. A tale of two deaths? Hepatology. 2006:43:31-44.

6. Borysova EO. Antihistamines: questions of security. Lechebnoe Delo. 2005;(2):37-43.

7. Drohovoz SM, Lukyanchuk VD, Sheyman BS, Kononenko AV. Toxic effect of histamine H1-receptor blockers and mechanisms of their formation. Modern Problems of Toxicology. 2012;3-4:44-48.

8. Kuzminov OB. Evaluation of immune toxic effects of loratadine on laboratory animals in the experiment. Experimental and Clinical Physiology and Biochemistry. 2014;1:43-46.

9. Sharon N. Lectins: carbohydrate-specific reagents and biological recognition molecules. J Biol Chem. 2007;282:2753-2764.

10. Gabius HJ (ed.). The sugar code: fundamentals of glycosciences. Weinheim: Wiley-Blackwell; 2009. p. 317-328.

11. Roth J. Lectins for histochemical demonstration of glycans. Histochem Cell Biol. 2011;136:117-130.

12. Dan X, Liu W, Ng TB. Development and applications of lectins as biological tools in biomedical research. Med Res Rev. 2015; DOI: 10.1002/med.21363.

13. Weakley BS. Electron microscopy for beginners. Moscow: Mir; 1975. p. 314.

14. Lutsyk A, Ambarova N, Antonyuk V. Diabetic alteration versus postnatal maturation of rat kidney glycoconjuga- tes: comparative detection by lectin probes. Folia Histochem Cytobiol. 2013;51(1):92-102.

15. Antonyuk VO. Lectins and their resources. Lviv: Kvart; 2005. p. 554

16. Dudok OV, Kovalyshyn VI, Lutsyk AD. Liver ultrastructure after administration of Loratadine. Acta Medica Leopoliensia. 2015;21(2):63-68.

17. Yvashkin VT, Nepomnyashchyh GN, Aydagulova SV. Drug-induced liver injury: Universal structural markers. Russian Journal of Gastroenterology, Hepatology, Coloproctology. 2009;12(2):20-29.

18. Kuznetsova LV. The role of liver in human immune system. Family Medicine. 2008;2:37-40.

19. Yakovleva LV, Lytvynenko GL, Loryanovska YB. Study of the most active derivatives among benzofuran derivatives (C 764-0334), 1.5 dyhidropirol-2-ones (VAZ-10) and 2,3-D-pyrimidine-6-carboxylic acid (L 486-0021) on liver function in healthy rats. Clinical Pharmacy, Pharmacotherapy and Medical Standardization. 2011;3-4:71-75.

20. Camelo-Nunes IC. New antihistamines: a critical view. J Pediatr (Rio J). 2006;82;5(Suppl.):173-180.

21. Berezhnaya NM, Kotova SA, Evseeva TA. Intracellular regulation of the functional state of lymphocyte $\mathrm{H} 1$ - and $\mathrm{H} 2$ - receptors in atopy - a possible criterion for individual selection of antihistamines. Allergology and Immunology. 2000;1(1):93-100.

22. Takeishi T, Mirano K, Kobayashi T et al. The role of Kupffer cells in liver regeneration. Arch Histol Cytol. 1999;62(5):413-422.

23. Deltsova OI, Gerashchenko SB, Kulinich GB. Stem cells and regeneration of liver. Scientific Bulletin of Uzhgorod University. 2012;1(43):175-179.

24. Yashchenko AM, Pankevych LV, Lutsyk AD. Rat liver carbohydrate alterations in streptozotocin-induced diabetic rats. Eur J Anat. 2012;16(2):82-90.

Acceptance for editing: 2016-06-29 Acceptance for publication: 2016-06-30

Correspondence address: Olga Dudok Department of Histology, Cytology and Embryology Danylo Halytsky Lviv National Medical University 69 Pekarska St, 79010 Lviv, Ukraine phone: + 380985505304 email: dudok.olga@gmail.com 